\title{
Recomendaciones de enfermería para un retorno seguro al trabajo ante COVID-19
}

\section{Nursing recommendations for a safe return to work against COVID-19}

\author{
María F. González Trejo ${ }^{a}$, Reyna C. Jiménez-Sánchez ${ }^{b}$, Rosario Barrera-Gálvez ${ }^{c}$, Esther \\ Ramírez-Moreno $^{d}$, María L. Sánchez-Padilla ${ }^{e}$, Rosa M. Baltazar-Téllez ${ }^{f}$, José Arias-Rico $^{g}$
}

\begin{abstract}
:
The objective of this work was to carry out a bibliographic and systematized search of works that propose general recommendations of preventive measures for a safe return to work in the face of COVID-19. This work is a descriptive and retrospective study. It was based on the active search for various sources of information in different databases. Due to the repercussions in the work that have been presented by COVID-19, it is necessary to establish strategies and implement actions to be carried out at the work centers, breaking the transmission chains in a timely manner, guaranteeing the continuity of the activity in their work centers. The recommendations for employers and workers in the safe return to work are based on a strategic plan that includes measures of: Hand washing, respiratory hygiene, education and training, as well as preventive measures in the journey from home to the center of work and vice versa (vehicle, hygiene, public transport) monitored and supervised to verify the correct implementation of all measures in companies or the workplace.
\end{abstract}

Keywords:

COVID-19, Employers, Plan, Job, Insurance

Resumen:

El objetivo de este trabajo fue el de hacer una busqueda bibliografica y sistematizada de trabajos que propongan recomendaciones generales de medidas preventivas para un retorno seguro al trabajo ante el COVID-19. Este trabajo es un estudio descriptivo y retrospectivo. Estuvo fundamentada en la búsqueda activa de diversas fuentes de información en diferentes bases de datos. Resultados y Discusión: Debido a las repercusiones en el trabajo que por el COVID-19 se han presentado, es necesario establecer estrategias e implementar acciones que permitan a los centros de trabajo, romper de manera oportuna las cadenas de trasmisión, garantizando la continuidad de la actividad en sus centros laborales. Las recomendaciones para los empleadores y trabajadores en el retorno al trabajo seguro se basan en un plan estratégico que incluye medidas de: Lavado de manos, higiene respiratoria, educación y capacitación, así como medidas preventivas en el trayecto de la casa al centro de trabajo y viceversa (Vehículo, higiene, transporte público) vigiladas y supervisadas para constatar la correcta implementación de todas las medidas en las empresas o el centro de trabajo.

Palabras Clave:

COVID-19, Empleadores, Plan, Trabajo, Seguro

\footnotetext{
${ }^{a}$ Universidad Autónoma del Estado de Hidalgo, https://orcid.org/0000-0001-5039-0439, Email: go352877@uaeh.edu.mx

${ }^{b}$ Universidad Autónoma del Estado de Hidalgo, https://orcid.org/0000-0001-9264-8514, Email: jimenezs@uaeh.edu.mx

${ }^{c}$ Universidad Autónoma del Estado de Hidalgo, https://orcid.org/0000-0002-1949-5424, Email: rosario_barrera@uaeh.edu.mx

${ }^{d}$ Universidad Autónoma del Estado de Hidalgo, https://orcid.org/0000-0002-9928-8600, Email: esther_ramirez@uaeh.edu.mx

${ }^{e}$ Universidad Autónoma del Estado de Hidalgo, https://orcid.org/0000-0002-2168-7564, Email: maria_sanchez2789@uaeh.edu.mx

${ }^{f}$ Universidad Autónoma del Estado de Hidalgo, https://orcid.org/0000-0003-2628-5932, Email: rosa_baltazar@uaeh.edu.mx

Autor de Correspondencia, Universidad Autónoma del Estado de Hidalgo, https://orcid.org/0000-0003-0219-0410, Email:
} jose_arias@uaeh.edu.mx 


\section{Introducción}

Como se sabe, la enfermedad del coronavirus 2019 (COVID-19) es una enfermedad respiratoria causada por el virus SARS-CoV-2, el cual se ha propagado desde China hacia todo el mundo. ${ }^{1,2,3}$ Es importante saber que una pandemia se presenta en 2 o 3 oleadas que separan a cada brote durante 3 a 9 meses. . $^{4,5}$ Durante una pandemia puede que se requiera que empresas, organizaciones sociales o escuelas tomen medidas únicas para ayudar a frenar la propagación de la enfermedad incluyendo el cierre por orden oficial de las autoridades de salud, además de otras medidas como limitar o cancelar reuniones sociales y públicas, reducir el flujo de transporte público, establecer cuarentenas en personas enfermas o que tuvieron contacto con una. ${ }^{6,7}$

La recuperación de estas situaciones puede no comenzar inmediatamente. Por lo que es importante asegurarse de que las actividades esenciales de cada negocio puedan mantenerse durante varias semanas 0 meses con personal limitado ${ }^{[8]}$, además cada empresa 0 negocio deberá estar preparado para reanudar actividades de una forma planificada que provea las condiciones necesarias que prevengan y/o retrasen la propagación de COVID-19 durante el regreso de los empleados a sus actividades. ${ }^{9,10}$

Tanto los empleadores, como el personal, deben estar capacitados para poder implementar un plan de acción para el regreso al trabajo en condiciones postepidémicas. ${ }^{11,12}$

Es por este motivo, que durante el regreso al trabajo y en nuestra vida cotidiana, debemos mantener las recomendaciones que las autoridades de salud nos han indicado hasta ahora: Uso del cubre bocas, lavado frecuente de manos, sana distancia, estornudo de etiqueta. ${ }^{13,14,15,16]}$

\section{Readaptación al trabajo}

Este virus puede presentarse con síntomas los cuales podrían hacernos creer que es una gripe normal y común sin embargo al detectar algún síntoma debemos tomar las medidas necesarias. Los estudios científicos han encontrado que el virus puede sobrevivir en las superficies desde unas horas hasta varios días.

Los síntomas más comunes son tos seca, cansancio y dolor muscular, aunque la persona afectada puede presentar signos más graves siendo fiebre de más de $38^{\circ}$, falta de aire y dificultad para respirar, así como también signos de menor frecuencia, dolor de cabeza, dolor de garganta, dolor abdominal y diarrea. El virus se propaga de una persona infectada a otra a través de gotas que expulsa una persona enferma al toser o estornudar , al tocar un objeto o superficie contaminada con el virus \& luego llevarse las manos sucias a boca, nariz u ojos. ${ }^{17}$

Aunque todos podemos contagiarnos de Covid19 hay una parte de la poblacion que requiere tener mayor cuidado, por el riesgo de presentar complicaciones, algunas son: personas mayores de 60 años, mujeres embarazadas, personas con enfermedades crónicas (obesidad, hipertensión, diabetes, enfermedad pulmonar obstructiva crónica, enfermedad cardiovascular.) entre otros.

En caso de pertenecer a alguno de estos grupos se debe de notificar a los jefes para que se evalue la posibilidad de realizar el trabajo desde casa o bien fortalecer las medidas de seguridad dentro del área de trabajo.

\section{Metodología}

El objetivo de este trabajo fue el de hacer una busqueda bibliografica y sistematizada de trabajos que propongan recomendaciones generales de medidas preventivas para un retorno seguro al trabajo ante el COVID-19.

Se trata de un estudio retrospectivo y descriptivo. La estrategia de investigación estuvo fundamentada en la búsqueda activa y manual de diversas fuentes de información en bases de datos como: Dialnet, Google Scholar, Scielo y Redalic. Se consideró tomar como principal fuente de información a aquellos documentos (guías nacionales e internacionales) que brindaron recomendaciones para la prevención de la transmisión e infección por COVID - 19 en centros laborales.

\section{Resultados y Discusión}

Se encontraron 26 bibliografías que proponen medidas de un retorno seguro al trabajo. Debido a las repercusiones en el Trabajo que por la COVID-19 se han presentado, es necesario establecer estrategias e implementar acciones que permitan a los centros de trabajo, romper de manera oportuna las cadenas de trasmisión, garantizando la continuidad de la actividad en sus centros laborales, aun cuando se presenten nuevos casos. $^{18}$

Se recomienda e informa al empleador y a los trabajadores sobre las medidas de etiqueta respiratoria y de higiene que se debe seguir en el lugar de trabajo. Dando facilidades para que estos puedan seguir laborando preferentemente desde casa e informar a los empleados que ciertas personas tienen un riesgo más alto de sufrir complicaciones de COVID-19. ${ }^{19}$

Es importante que las diferentes instituciones lleven a cabo las medidas de seguridad dadas por el Gobierno de México y La Secretaria de Salud de México. ${ }^{20 \text {, }}$ 21, 22. 23, 24 
- Verificar el establecimiento de las medidas de prevención y protección en el centro de trabajo.

- $\quad$ Verificar la provisión constante de agua, jabón y toallas desechables, y de soluciones a base de alcohol gel al $60-70 \%$ en todas las áreas de las empresas y centros de trabajo.

- Vigilar las disposiciones que establezcan las autoridades competentes para las posibles modificaciones de las acciones a seguir en el centro de trabajo.

- - Llevar el registro y seguimiento del personal en resguardo domiciliario voluntario y evaluar posibles casos de contagio.

- Crear un mecanismo de seguimiento de personas trabajadoras en aislamiento y, en caso de ser necesario, contactar a la autoridad sanitaria estatal.

Medidas preventivas en el trayecto de la casa al centro de trabajo y viceversa:

- Limpiar y desinfectar las áreas y objetos del interior del auto, poniendo especial atención en el volante, manijas y palanca de velocidades.

- La limpieza se puede hacer con un paño con jabón y otro húmedo o utilizar un paño con solución de hipoclorito de sodio comercial (diluir $100 \mathrm{ml}$ de la solución comercial en $900 \mathrm{ml}$ de agua).

- Al ingresar o subir a cualquier transporte público utilizar cubre bocas. Se deberá colocar asegurando su correcto ajuste al contorno de la cara.

- Procurar sana distancia con el resto de los pasajeros.

- Tratar de no tocar nada que no sea necesario.

- No tocar la cara en todo momento hasta que se haya realizado higiene de manos.

- $\quad$ Si se mueve el cubre bocas y hay necesidad de ajustarlo, antes de hacerlo primero desinfectar las manos con alcohol gel (alcohol al 60-70\%). Una vez hecho el ajuste, volver a desinfectar las manos.

- Si durante el retorno a casa se estuvo en contacto con varias personas o en lugares donde no se pudo cuidar la sana distancia, se sugiere tomar un baño una vez que se ingrese al hogar y antes de tener contacto con algún miembro de la familia.

- De no realizar el baño, se sugiere cambio de ropa de calle por una limpia, previo lavado de manos y/o desinfección con alcohol gel al 70\%.
- La ropa utilizada dejarla con la ropa sucia.

- Limpiar los objetos que pueden estar contaminados como la bolsa, llaves, portafolios, etc.

\section{Conclusiones}

Es importante promover las acciones que indican los sectores de salud e implementarlas para evitar que sigan aumentando los casos por COVID-19 y cuidarnos.

Conservar las manos limpias es una de las medidas más esenciales que podemos tomar para evitar enfermarnos y transmitir los microbios a otras personas. Sinfín de enfermedades y afecciones se propagan por no lavarse las manos con agua corriente limpia y jabón. Los CDC da a conocer que lavarse las manos de una manera específica para evitar enfermarse y propagar microbios a otras personas. Los resultados indican que lavarse las manos durante alrededor de 15 a 30 segundos elimina más microbios de las manos que lavárselas durante periodos más cortos. Por tanto, se debe de:25, 26

- Promover el lavado regular y minucioso de manos por parte de empleados, contratistas \& clientes.

- Colocar dispensadores de desinfectante para manos en lugares destacados del lugar de trabajo, lavarlos \& rellenarlos continuamente.

- Poner a la vista carteles que promuevan el lavado de manos.

- Trabajar con los médicos de empresa y líderes sindicales, para llevar a cabo sesiones informativas sobre las medidas que se están tomando.

- Garantizar que el personal tenga acceso a lugares en donde puedan lavarse las manos con agua y jabón.

Los siguientes son algunos momentos clave en los que los empleados deben lavarse las manos: ${ }^{26}$

1. Antes de iniciar y al finalizar los turnos de trabajo

2. Antes y después de los descansos en el trabajo

3. Después de sonarse la nariz, toser o estornudar

4. Después de ir al baño

5. Antes de comer o preparar comidas

A fin de prevenir el contagio respiratorio por covid-19 se debe de implementar una buena promoción de higiene respiratoria en el lugar de trabajo. El publicar en las entradas alertas visuales permite un mayor alcance pues los trabajadores lo visualizaran y permitirá focalizar las acciones que se estarán llevando acabó. Las siguientes 
medidas deberán de estar incluidas en las precauciones estándar del control de la infección.

- Exhibir carteles que promuevan la higiene respiratoria.

- Capacitar al personal de seguridad y de salud ocupacional, para difundir estas medidas.

- Proporcionar máscaras, pañuelos y desinfectantes para manos a base de alcohol convenientemente ubicados para áreas de espera y áreas de evaluación.

Brindar a los trabajadores educación y capacitación actualizadas sobre cómo se transmite y los factores de riesgo de Coronavirus, conductas protectoras e instrucciones sobre conductas adecuadas (por ejemplo: etiqueta para la tos: evitar tocarse la nariz, boca y ojos).

Proporcionar materiales de educación y capacitación en un formato fácil de entender y en el nivel apropiado de lenguaje y alfabetización para todos los empleados.

Dotar de equipo de protección personal, esto si es necesario para mantener a los trabajadores seguros mientras realizan su trabajo. El equipo es: cubre bocas N95, protectores faciales, gafas y guantes desechables. Esto en los trabajos de muy alto riesgo. El uso de cubre bocas ya es obligatorio en cualquier espacio comunitario.

\section{Referencias}

[1] Lai, C. C., Shih, T. P., Ko, W. C., Tang, H. J., \& Hsueh, P. R. Severe acute respiratory syndrome coronavirus 2 (SARS-CoV-2) and corona virus disease-2019 (COVID-19): the epidemic and the challenges. International journal of antimicrobial agents, [Internet]. 2020 [Citado 24 oct 2020 ]105924. Disponible en: https://doi.org/10.1016/j.ijantimicag.2020.105924

[2] Ahn, D. G., Shin, H. J., Kim, M. H., Lee, S., Kim, H. S., Myoung, J., ... \& Kim, S. J. Current status of epidemiology, diagnosis, therapeutics, and vaccines for novel coronavirus disease 2019 (COVID-19). Journal of microbiology and biotechnology[Internet]. 2020 [Citado 24 oct 2020 ] 30(3), 313-324. Disponible en: https://europepmc.org/article/med/32238757

[3] Boulos, M. N. K., \& Geraghty, E. M. Geographical tracking and mapping of coronavirus disease COVID-19/severe acute respiratory syndrome coronavirus 2 (SARS-CoV-2) epidemic and associated events around the world: how 21st century GIS technologies are supporting the global fight against outbreaks and epidemics. International Journal of Health Geographics[Internet]. 2020 [Citado 25 oct 2020] 19: 8. Disponible en: https://www.ncbi.nlm.nih.gov/pmc/articles/PMC7065369/

[4] Tomasi, S. N. HISTORIA DE LAS PANDEMIAS MUNDIALES Y LA ECONOMIA. Academia[Internet] 2020 [Citado 25 oct 2020]. Disponible en: https://cutt.ly/YgETGT7

[5] Amadeo P. Sopa de Wuhan, pensamiento contemporáneo en tiempos de pandemias. [Internet] 2020 [citado 25 oct 2020]. Disponible en: https://cutt.ly/igETKgm
[6] Pan, S. L., Cui, M., \& Qian, J. Information resource orchestration during the COVID-19 pandemic: A study of community lockdowns in China. International Journal of Information Management, [Internet]. 2020 [Citado 25 oct 2020] 102143. Disponible en: https://www.ncbi.nlm.nih.gov/pmc/articles/PMC7211621/

[7] Gostin, L. O., \& Wiley, L. F. Governmental public health powers during the COVID-19 pandemic: stay-at-home orders, business closures, and travel restrictions. Jama Network, [Internet]. 2020 [Citado 25 oct 2020] 323(21), 2137-2138. Disponible en: https://jamanetwork.com/journals/jama/fullarticle/2764283

[8] Diez Canseco Terry, R., Salas, R., Vásquez, E., Alberto Valcárcel, L., Butler, M., Stoessel, J., ... \& Torres Paz, L. Reinventando el Turismo en tiempos del COVID-19. Universidad San Ignacio de Loyol. [Internet] 2020 [Citado 25 oct 2020]. Disponible en: http://repositorio.usil.edu.pe/handle/USIL/9891

[9] Robles, A. La seguridad y continuidad de los negocios como efecto dinamizador para los destinos en la etapa pos-COVID. Turismo posCOVID-19. Reflexiones, retos y oportunidade Dialnet. [Internet] 2020 [Citado 25 oct 2020] ISBN 978-84-09-21816-5, págs. 107-116. Disponible en:

https://dialnet.unirioja.es/servlet/articulo?codigo $=7506833$

[10] Koonin, L. M. Novel coronavirus disease (COVID-19) outbreak: Now is the time to refresh pandemic plans. Journal of business continuity \& emergency planning, [Internet] 2020 [Citado 26 oct 2020]13(4), 1-15. Disponible en: https://pubmed.ncbi.nlm.nih.gov/32160942/

[11] Barnes, M., \& Sax, P. E. Challenges of "Return to Work" in an ongoing pandemic. The New England Journal of Medicine. [Internet] 2020 [Citado 26 oct 2020]. Disponible en: https://www.nejm.org/doi/pdf/10.1056/NEJMsr2019953?articleTools $=$ true

[12] Taylor, T., Das, R., Mueller, K., Pransky, G., Christian, J., Orford, R., \& Blink, R. Safely Returning America to Work Part I: General Guidance for Employers. Europe PMC[Internet] 2020 [Citado 26 oct 2020] PMID: 32858558. Disponible en: https://europepmc.org/article/med/32858558\#impact

[13] Levy, T. S., Acosta, L. M. G., Rosas, V. M., Nasu, L. C., Pineda, E. B. G., Arcos, M. A. A., ... \& Dommarco, J. A. R. Ensars-cov-2. Resultados de la evaluación basal de la encuesta nacional de las características de la población durante la pandemia de covid-19. Google Académico. [Internet] 2020 [Citado 26 oct 2020]. Disponible en: $\underline{\text { htps: } / / \mathrm{n} 9 . \mathrm{cl} / \mathrm{xgv} 94}$

[14] Patiño Fierro, M. P., \& Cruz Reyes, G. Las medidas adoptadas por las entidades federativas ante la emergencia del Covid-19. Instituto Belisario Domínguez [Internet] 2020 [Citado 26 oct 2020] Disponible en: http://bibliodigitalibd.senado.gob.mx/handle/123456789/4856

[15] Stone, T. E., Kunaviktikul, W., Omura, M., \& Petrini, M. Facemasks and the Covid 19 pandemic: What advice should health professionals be giving the general public about the wearing of facemasks?. Nursing \& Health Sciences. [Internet] 2020 [Citado 26 oct 2020] Disponible en: https://onlinelibrary.wiley.com/doi/epdf/10.1111/nhs.12724

[16] Freeman, S., \& Eykelbosh, A. COVID-19 and outdoor safety: Considerations for use of outdoor recreational spaces. National Collaborating Centre for Environmental Health. [Internet] 2020 [citado 26 oct 2020]. Dispónible en: https://n9.cl//ru1

[17] Barboto Salazar, B. A. Proceso de atención de enfermería en paciente de 66 años con VIH y Neumonía asociada a Covid-19. 
Publicación semestral, Educación y Salud Boletín Científico Instituto de Ciencias de la Salud Universidad Autónoma del Estado de

Hidalgo, Vol. 9, No. 18 (2021) 28-32

Bachelor's thesis, Babahoyo: UTB-FCS[Internet], 2020 [Citado 26 oct

2020]. Disponible en: http://dspace.utb.edu.ec/handle/49000/8279

[18] Méndez Fuentes L. A., Mata X. Manual para el retorno saludable, seguro y confiable al trabajo. Asociación mexicana de distribuidores de automotores A.C. [Internet] 2020 [Citado el 28 octubre 2020], Disponible en: https://www.amda.mx/wp-content/uploads/ProtocoloCovid-19.pdf

[19] Gobierno de México, \& Secretaría de Salud México.

Recomendaciones para un retorno Seguro al Trabajo COVID 19. [Internet]. México: Gobierno de México, \& Secretaría de Salud México: 2020. [Citado 28 oct 2020]. Disponible en: https://n $9 . \mathrm{cl} / 47 \mathrm{v} 4 \mathrm{c}$

[20] Gobierno de México, \& Secretaría de Salud México. ACUERDO por el que se establecen acciones extraordinarias para atender la emergencia sanitaria generada por el virus SARS-CoV2. Diario Oficial. [Internet]. 31 mar 2020 [citado 28 octubre 2020]. Disponible en: https://n9.cl/4sw2

[21] Gobierno de México. Lineamientos técnicos de seguridad sanitaria en el entorno laboral. Gobierno de México. [Internet] 2020 [citado 29 octubre 2020]. Disponible en:

http://nuevanormalidad.gob.mx/files/LT-SS-EL-2020.pdf?

[22] Gobierno de México, \& Secretaría de Salud México. ACUERDO por el que se establecen los Lineamientos Técnicos Específicos para la Reapertura de las Actividades Económicas. Diario official [Internet]. 29 may 2020 [citado 28 octubre 2020]. Disponible en: https://n9.cl/z159v

[23] Department of Labor USA, \& Department of Health \& Human Services USA. . Guía sobre la Preparación de los Lugares de Trabajo para el virus COVID-19. Retrieved [Internet] 2020 [Citado 26 oct 2020]. Disponible en: https://www.osha.gov/Publications/OSHA3992.pdf

[24] Gobierno de México, \& Secretaría de Salud México. COVID-19. [Internet]. México: Gobierno de México, \& Secretaría de Salud México: May 1, 2020. [Citado 26 oct 2020]. Disponible en: https://coronavirus.gob.mx/

[25] Norma Oficial Mexicana NOM-035-STPS-2018, Factores de riesgo psicosocial en el trabajo-Identificación, análisis y prevención [Internet] México: Secretaria de Salud: 2018. [Citado 26 oct 2020]. Disponible en: https://cutt.ly/ngEIRJm

[26] El lavado de manos. Centro para el control y prevención de enfermedades. [Internet] 2020 [Citado 29 octubre 2020]. Disponible en: https://n9.cl/r2ze 